\title{
In Vivo Tracking of Mesenchymal Stem Cell-Derived Extracellular Vesicles Improving Mitochondrial Function in Renal Ischemia-Reperfusion Injury
}

Hongmei Cao, ${ }^{\dagger, \S, \#}$ Yuanqiu Cheng, ${ }^{\dagger, \#}$ Heqi Gao, ${ }^{\star}$ Jie Zhuang, ${ }^{\dagger}$ Weiguang Zhang, ${ }^{\S}$ Qiang Bian, $"$ Fang Wang, ${ }^{\perp}$ Yuan Du, ${ }^{\perp}$ Zongjin Li, ${ }^{\dagger}$ Deling Kong, ${ }^{*}$ Dan Ding,,$\stackrel{*}{*}$ Yuebing Wang ${ }^{*} \dagger, \S$

${ }^{\dagger}$ Department of Biochemistry, Nankai University School of Medicine, Tianjin 300071, China

$\$$ The Key Laboratory of Bioactive Materials, Ministry of Education, The College of Life Sciences, Nankai University, Tianjin 300071, China

${ }^{\S}$ Department of Nephrology, Chinese PLA General Hospital, Chinese PLA Institute of Nephrology, State Key Laboratory of Kidney Diseases, Beijing 100853, China

${ }^{\perp}$ Tianjin Nankai Hospital, Tianjin 300100, China

${ }^{\|}$National Pesticide Engineering Research Center, Nankai University, Tianjin 300071, China

${ }^{\#}$ H.C. and Y.C. contributed equally to this work.

Corresponding Authors:

*Email: wangyuebing@nankai.edu.cn

*Email: dingd@nankai.edu.cn 


\section{Experimental Section}

Flow Cytometric Characterization of hP-MSCs: The hP-MSCs were dissociated with $0.25 \%$ trypsin-EDTA (Gibco, Grand Island, NY) and then washed with PBS containing $2 \%$ FBS. Afterwards, the cell suspensions were incubated with fluorescence conjugated antibodies including CD105 and CD90 (Abcam, Cambridge, MA) or unstained control for 30 mins at room temperature. Then the cells were washed with PBS and resuspended in FACS buffer. The FACS analysis was performed using a FACS Calibur ${ }^{\mathrm{TM}}$ flow cytometer (BD Biosciences), and the data were analyzed using the Cell Quest Pro software (BD Biosciences).

Glycerol-Induced AKI Mouse Model: The glycerol-induced AKI mouse model was set up as previously described. ${ }^{1}$ Briefly, hypertonic glycerol ( $8 \mathrm{ml} / \mathrm{kg}$ body weight of $50 \%$ glycerol solution) was intramuscular injected into the inferior hind limbs of male FVB mice (8-10 weeks old). Intramuscular injection of glycerol induces myelitis and hemolysis that caused toxic and ischemic tubular injury.

In Vivo Tracking of the MSC-EVs in Glycerol-Induced AKI Mice: AKI mice were randomly assigned into AIEgen $(n=5)$ or AIE-EV groups $(n=5)$. On day 3 after glycerol administration, a total of $80 \mu \mathrm{g}$ AIE-EVs resuspended in $100 \mu \mathrm{L}$ PBS was injected through the tail vein. The IVIS Lumina imaging system was utilized by placing the anesthetized mouse on the equipped platform $\left(\lambda_{\mathrm{ex}}=488 \mathrm{~nm}\right.$, signal collection: 500 to $700 \mathrm{~nm}(10 \mathrm{~nm}$ step $)$, exposure time $=200 \mathrm{~ms}$, scans: $2 \mathrm{~h}$, $12 \mathrm{~h}, 24 \mathrm{~h}, 36 \mathrm{~h}, 48 \mathrm{~h}$ and $72 \mathrm{~h}$ post-injection, respectively). The intensity of the fluorescent signals was quantified by average radiance from a fixed-area ROI over the kidney. Furthermore, ex vivo imaging was performed on $2 \mathrm{~h}, 24 \mathrm{~h}$ and $72 \mathrm{~h}$ post-injection. 


\section{REFERENCE}

(1) Bruno, S.; Grange, C.; Deregibus, M. C.; Calogero, R. A.; Saviozzi, S.; Collino, F.; Morando, L.; Busca, A.; Falda, M.; Bussolati, B.; Tetta, C.; Camussi, G. Mesenchymal Stem

Cell-Derived Microvesicles Protect against Acute Tubular Injury. J. Am. Soc. Nephrol. 2009, 20, 1053-1067. 


\section{Supplemental Result}
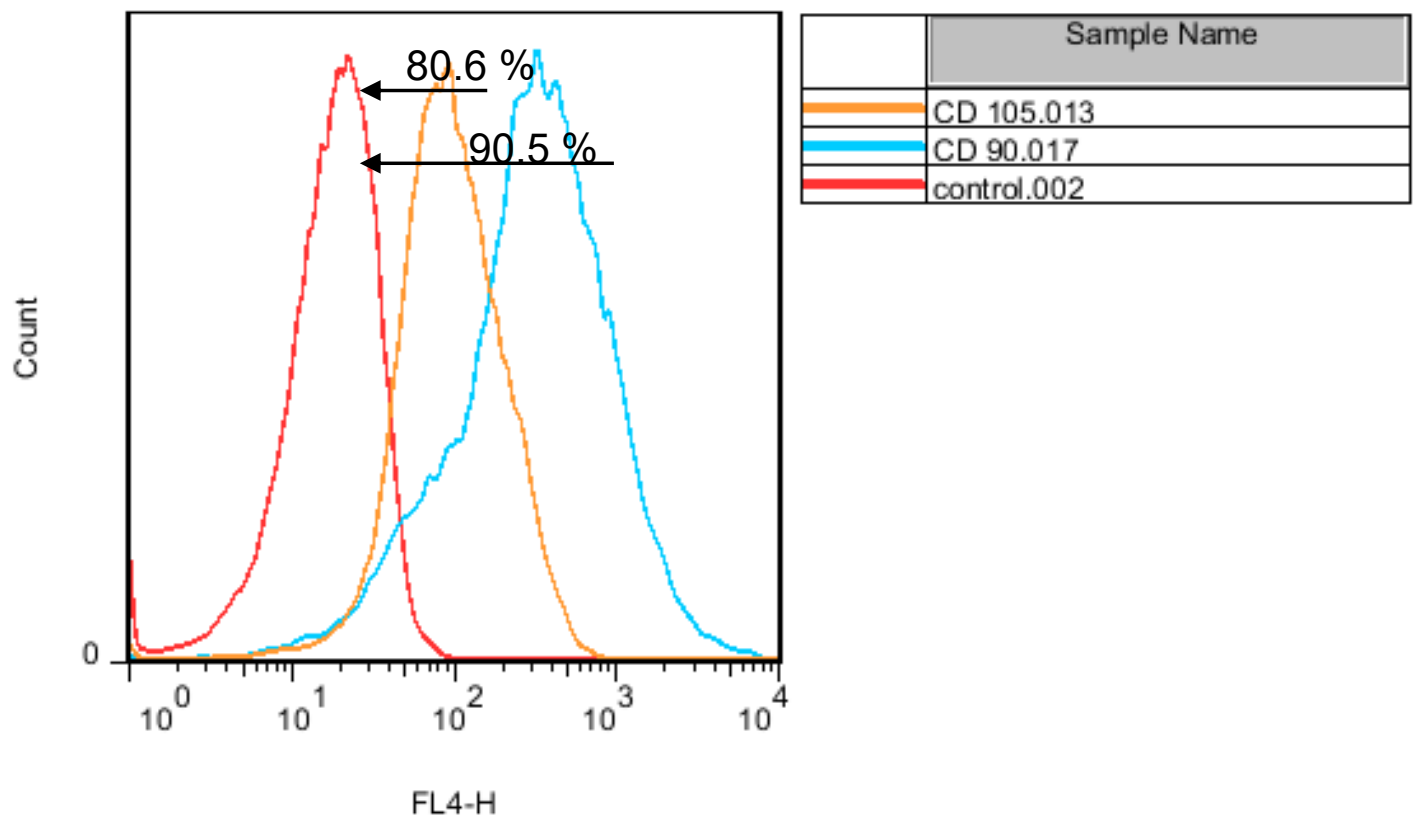

Figure S1. Phenotypic characterization of hP-MSCs by flow cytometry. Orange: CD105; Blue: CD90; Red: Control. 


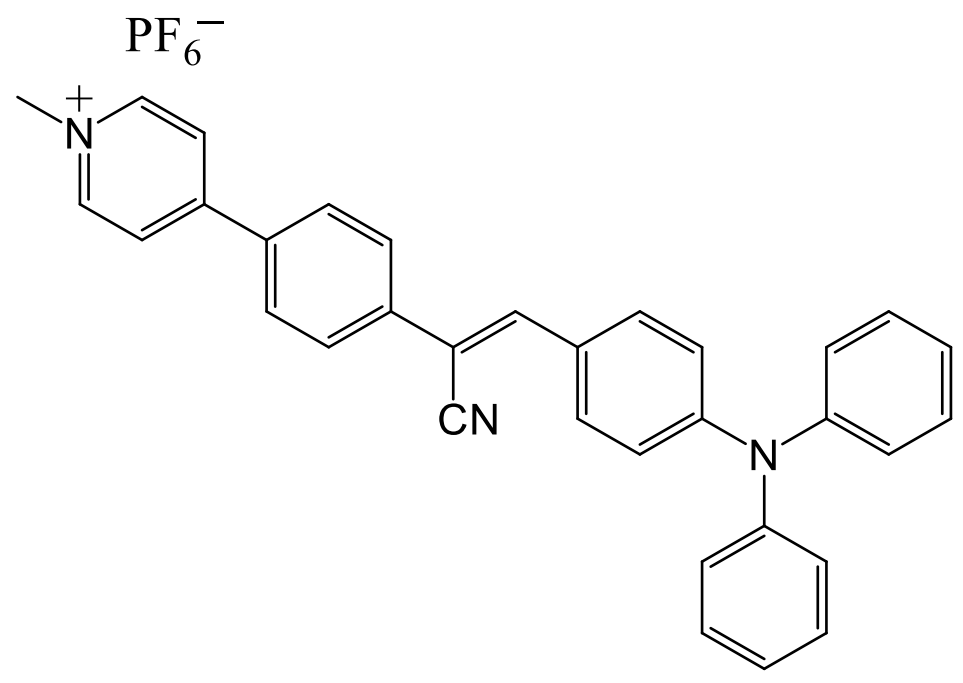

Figure S2. Chemical structure of DPA-SCP. 


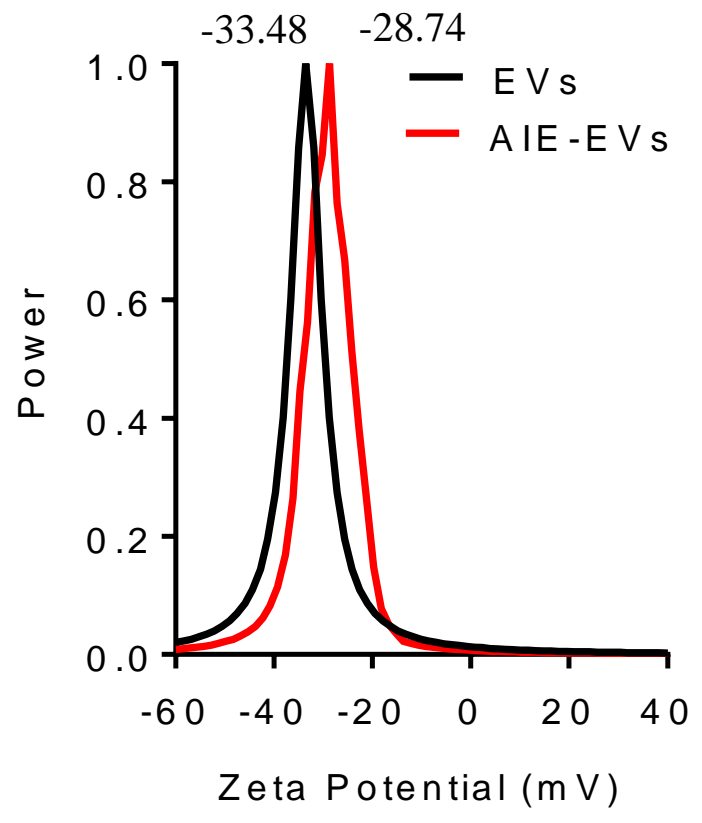

Figure S3. DLS measure of zeta-potentials for EVs and AIE-EVs. 


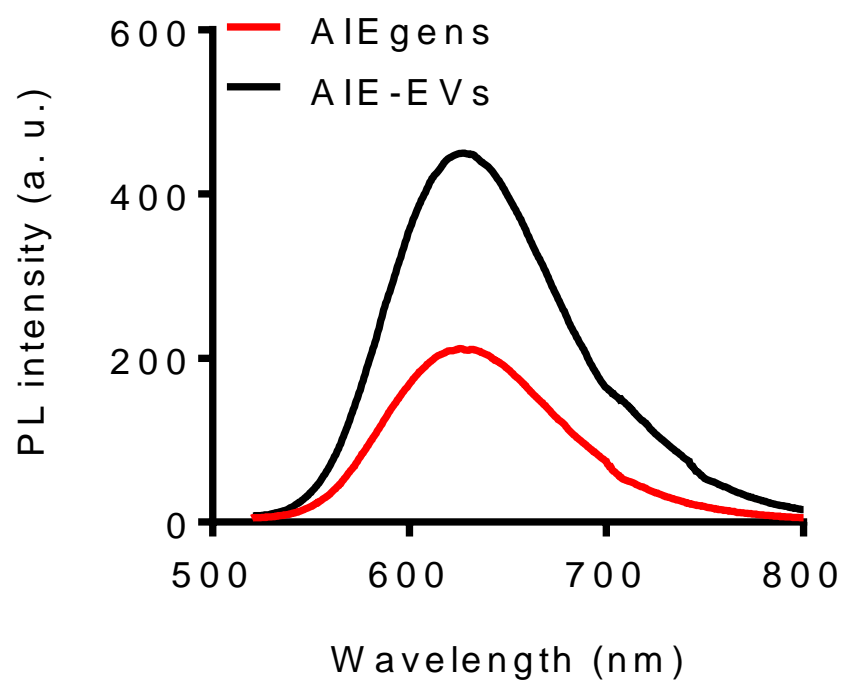

Figure S4. Photoluminescence spectra of AIEgens or AIE-EVs in PBS. 


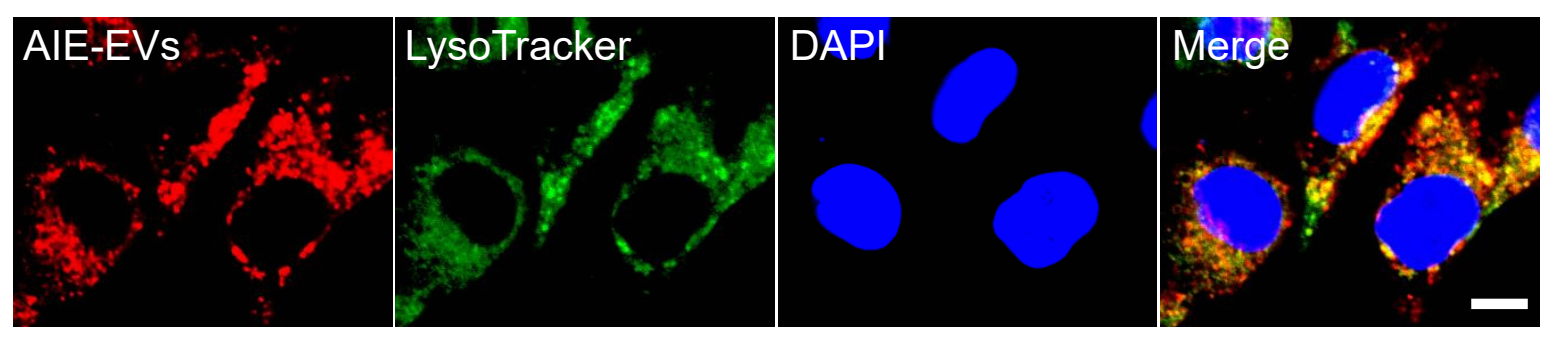

Figure S5. CLSM analysis of the eventual sorting of internalized AIE-EVs in TECs by LysoTracker staining. Red: AIE-EVs; Green: LysoTracker; Blue: DAPI. Scale bar, $10 \mu \mathrm{m}$. 


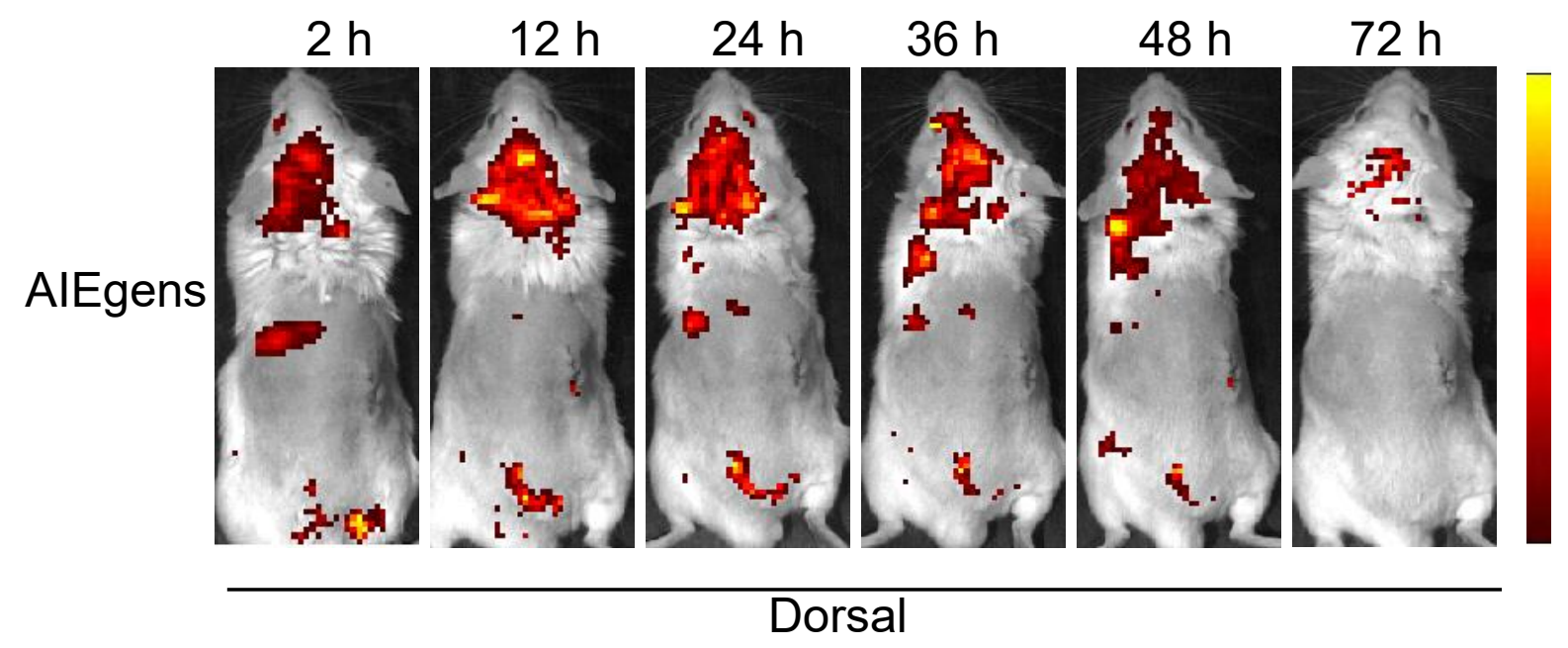

Figure S6. Representative fluorescence images of AKI mice after treatment with AIEgens. 


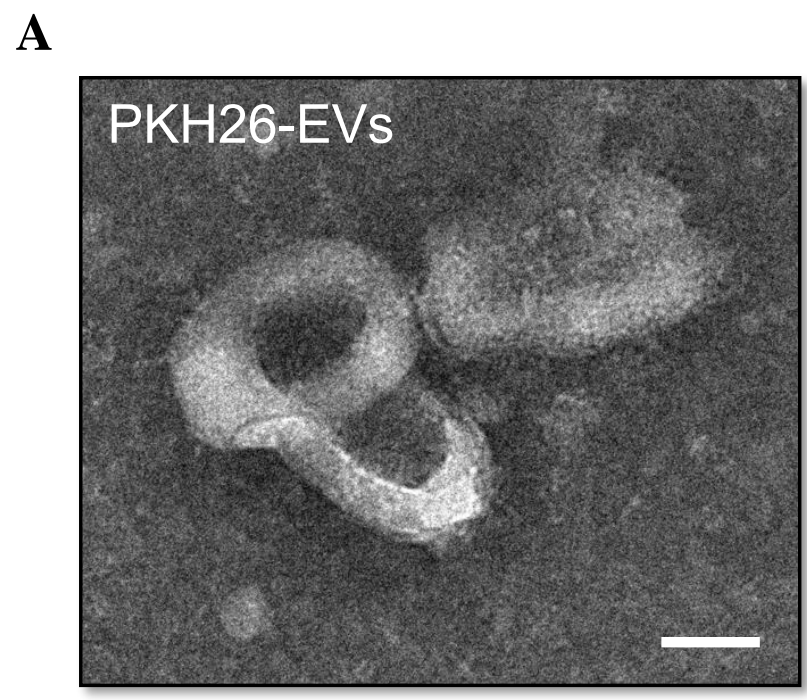

B

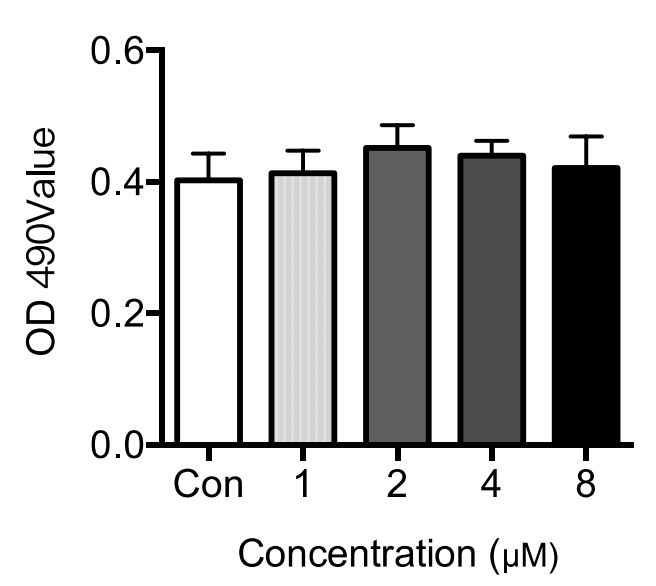

C

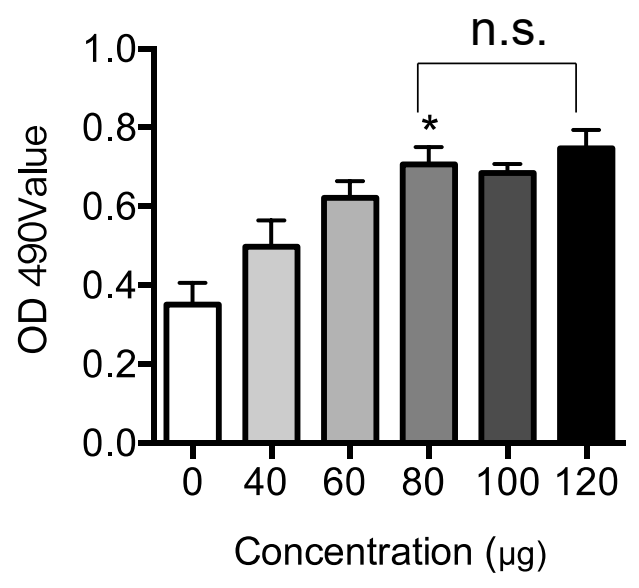

Figure S7. (A) TEM image of PKH26-EVs. Scale bar, $100 \mathrm{~nm}$. (B)CCK-8 assay assessing the cell toxicity of PKH26. (C) CCK-8 assay assessing the proliferation of TECs treated with different concentrations of PKH26-EVs ( $* P<0.05$ versus control; $n=3)$. 

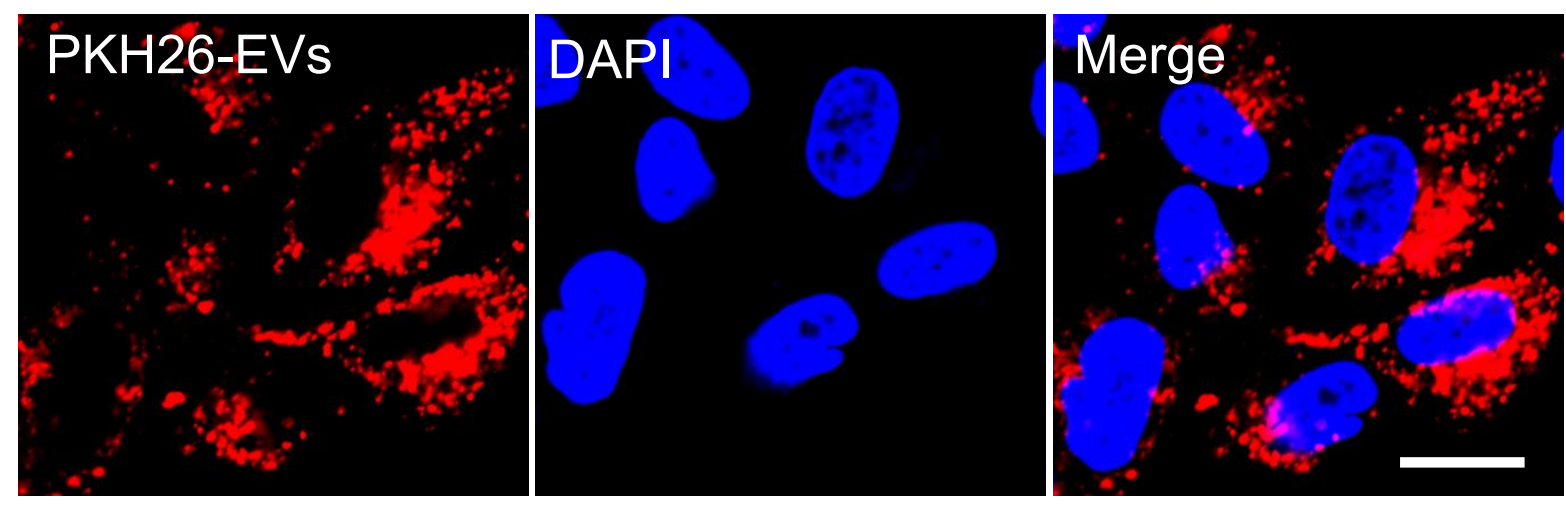

Figure S8. CLSM images of internalized PKH26-EVs. Red: PKH26- EVs; Blue: DAPI. Scale bar, $20 \mu \mathrm{m}$. 
A

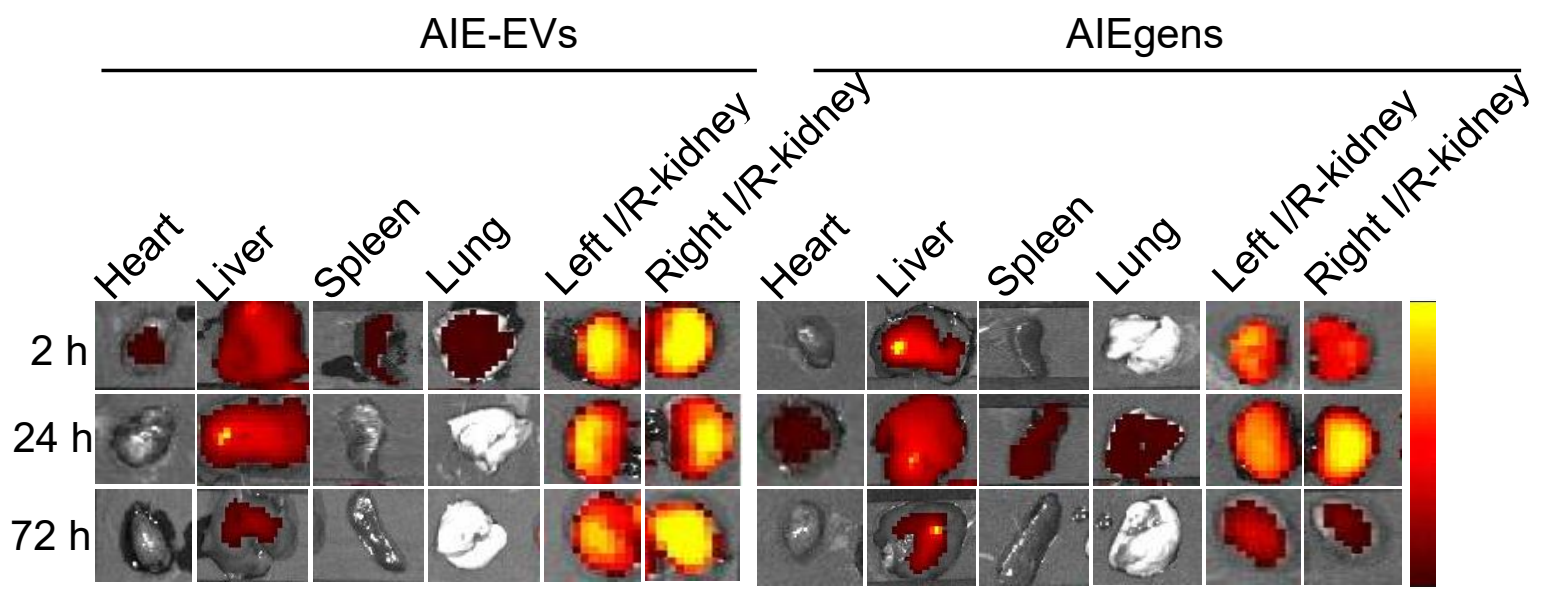

B

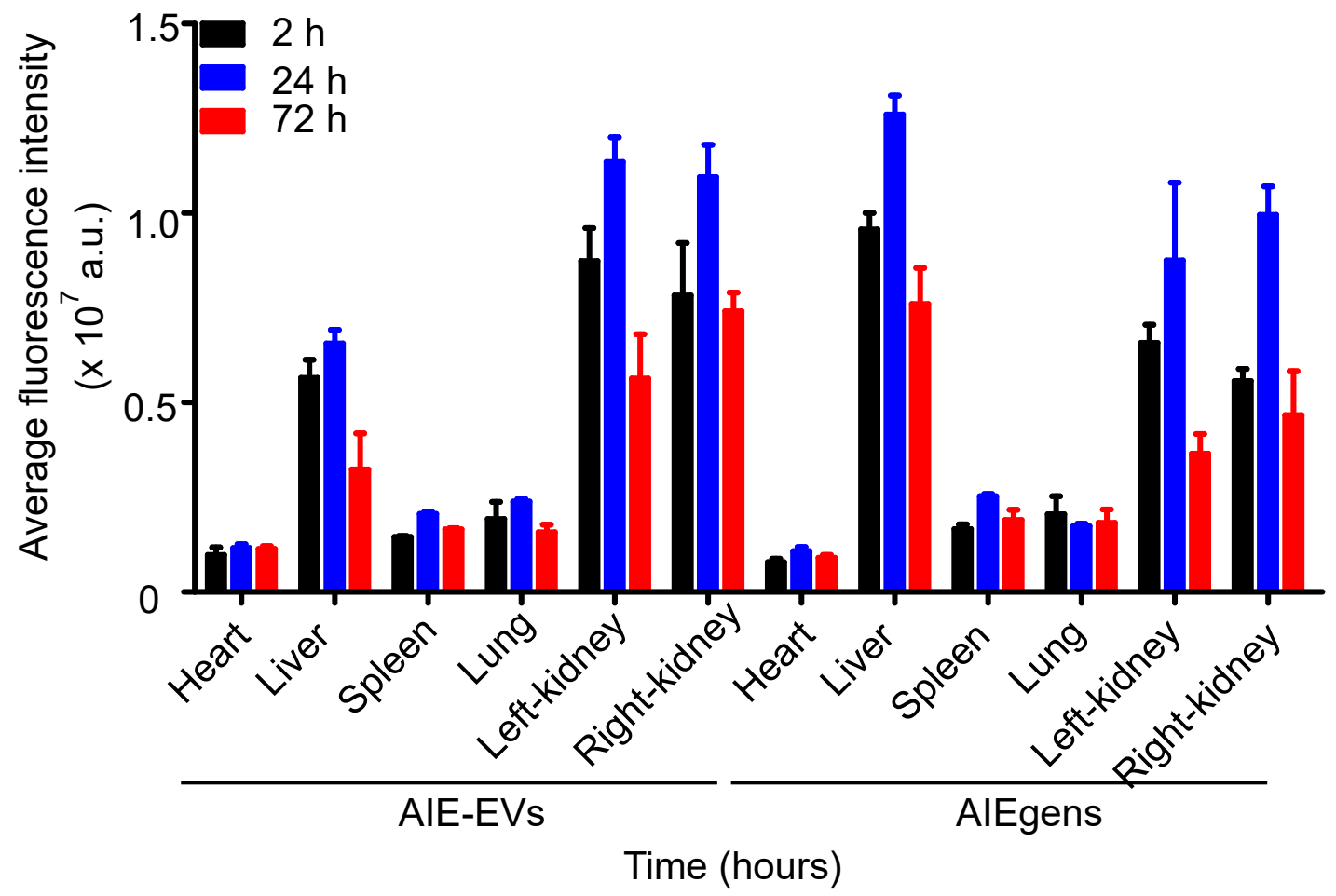

Figure S9. (A) Ex vivo fluorescence biodistribution of vital organs in glycerol-induced AKI mice.

(B) Time-dependent fluorescence intensity changes in vital organs at designated time intervals after sacrificing the glycerol-induced AKI mice $(n=5)$. 


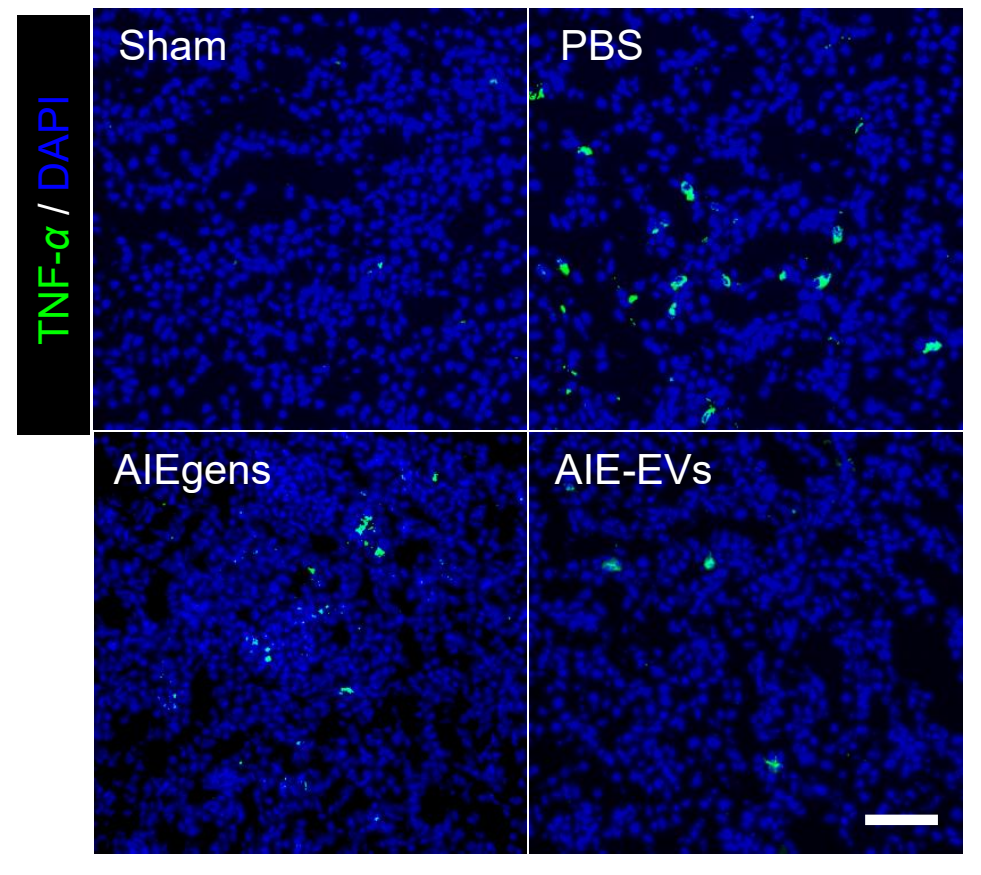

Figure S10. Representative TNF- $\alpha$ immunostaining in kidney tissues on day 7 postsurgery. Green: TNF- $\alpha$; Blue: DAPI. Scale bar, $50 \mu \mathrm{m}$. 
TNF- $\alpha$
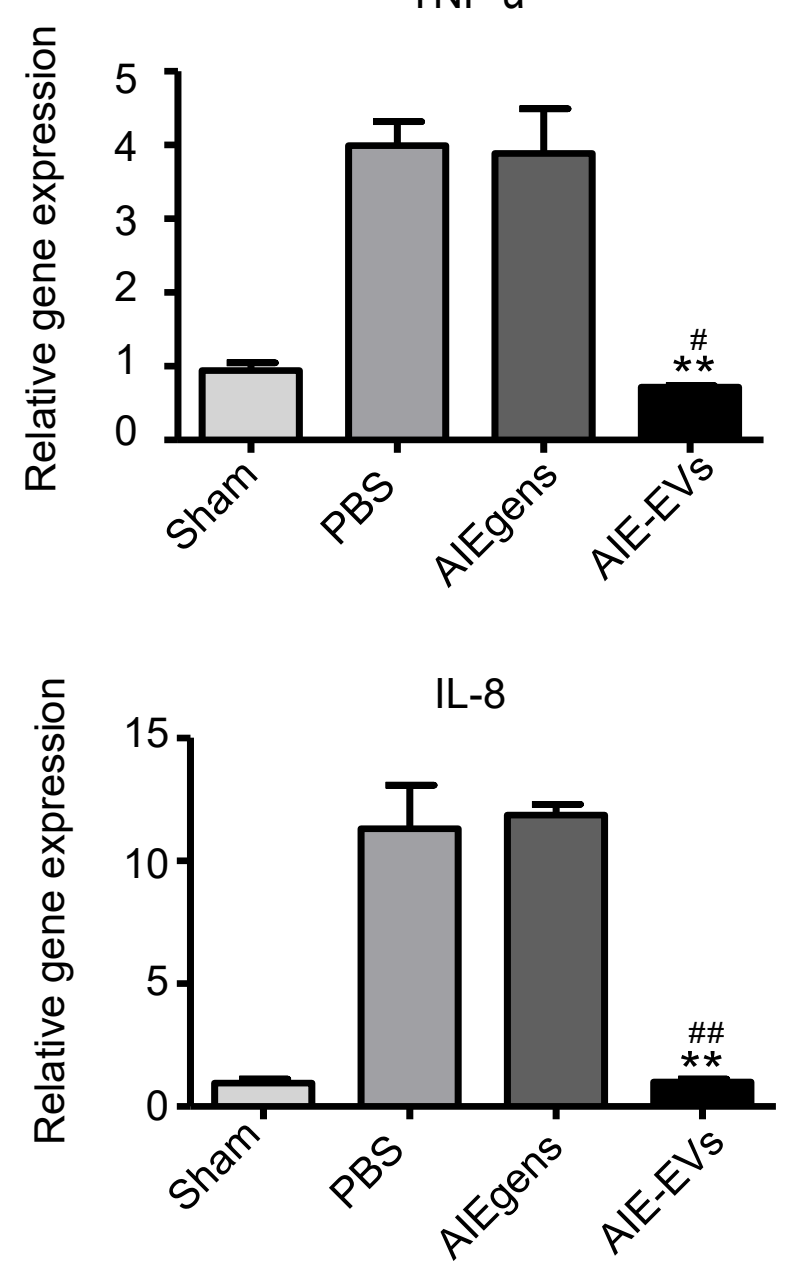
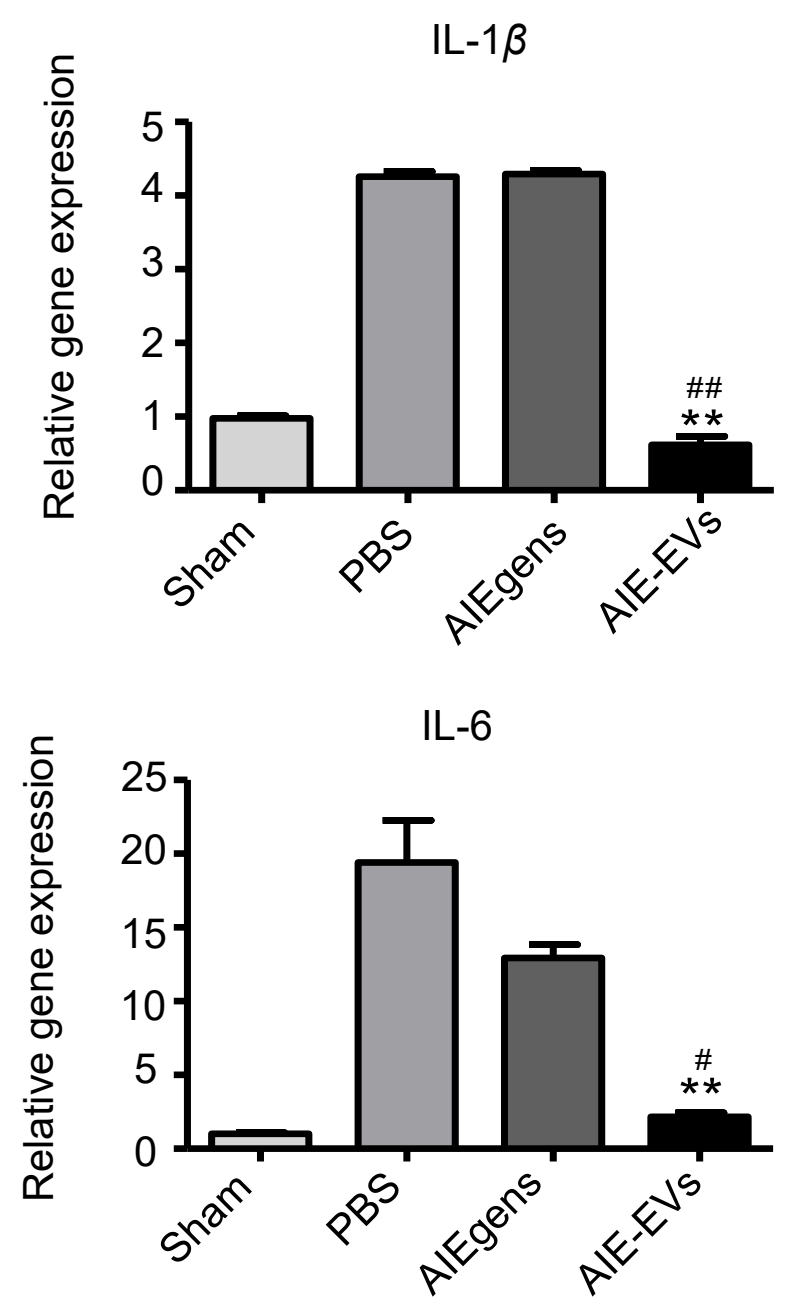

Figure S11. Detection of the pro-inflammatory gene expression by RT-qPCR analyses (** $P<$ 0.01 versus $\mathrm{PBS} ; \# P<0.05$ versus AIEgens; \#\# $P<0.01$ versus AIEgens; $n=5$ ). Each experiment was performed in triplicate. 


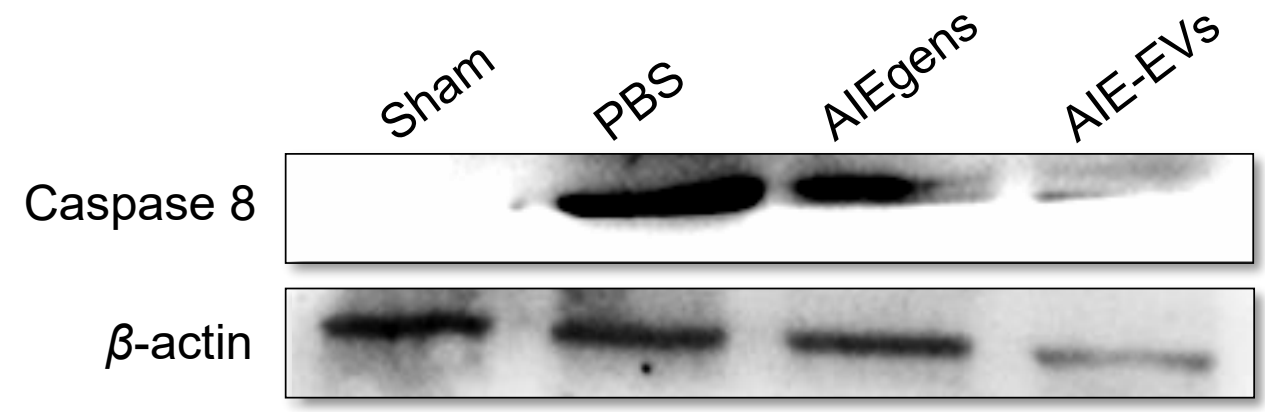

Figure S12. Western blot of Caspase 8 expression in kidney tissues from each group. Each experiment was performed in triplicate. 

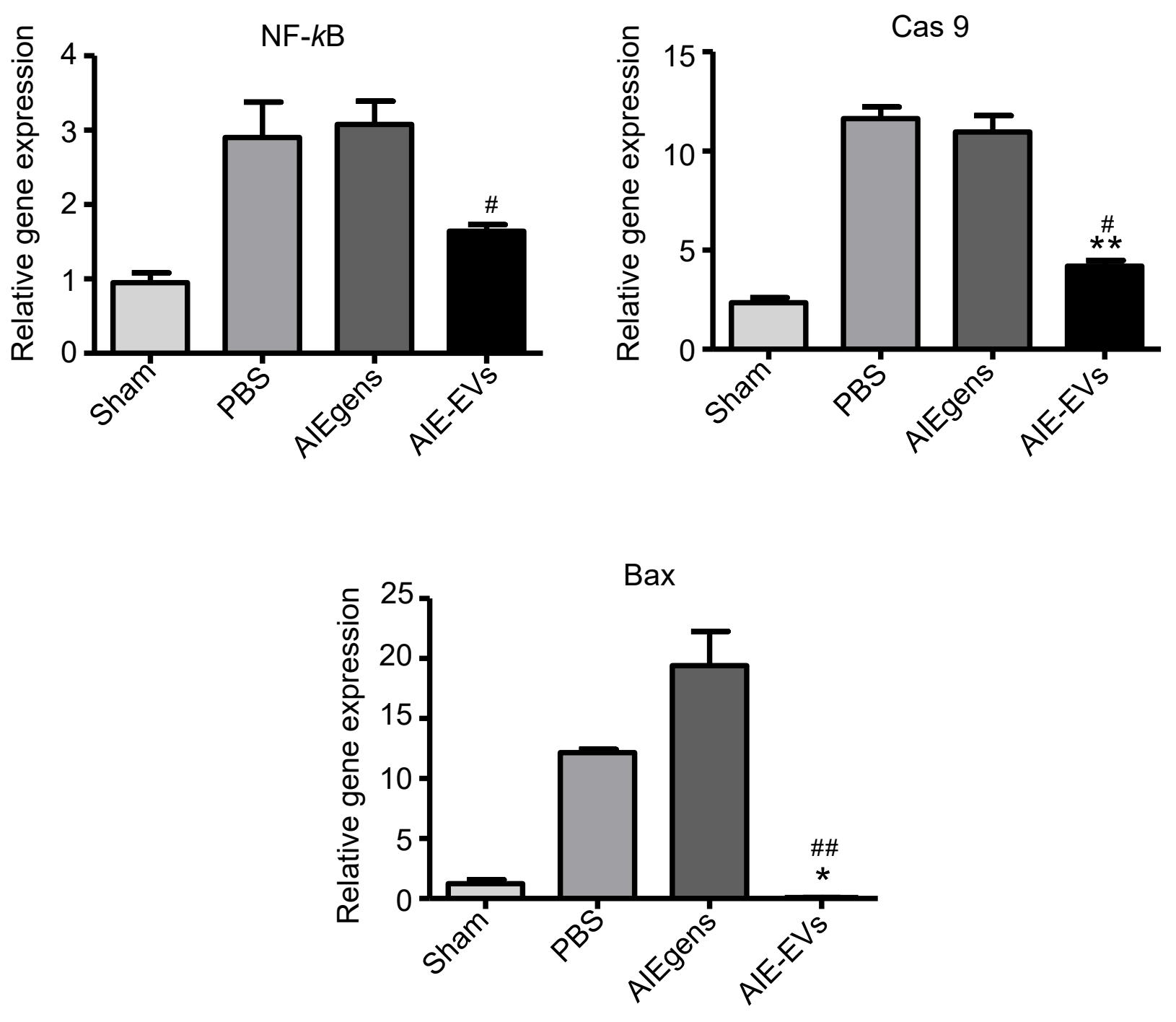

Figure S13. Detection of apoptosis-related gene expression by RT-qPCR analyses $(* P<0.05$ versus $\mathrm{PBS} ; * * P<0.01$ versus $\mathrm{PBS} ; \# P<0.05$ versus AIEgens; \#\# $P<0.01$ versus AIEgens; $n$ $=5$ ). Each experiment was performed in triplicate. 


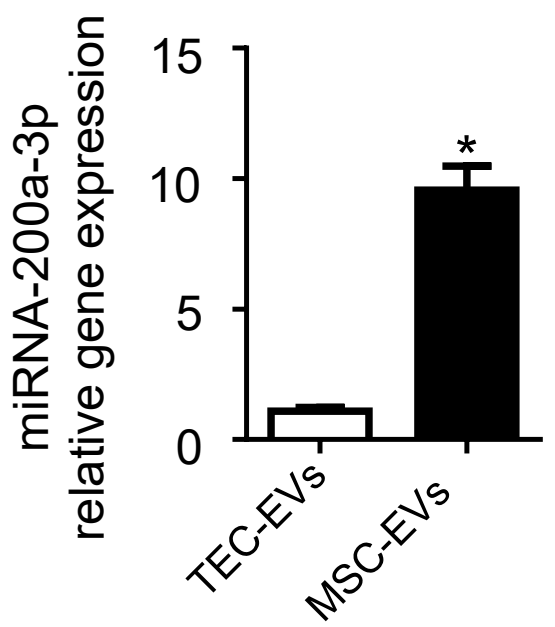

Figure S14. The expression levels of miRNA-200a-3p in MSC-EVs and TEC-EVs assessed by RT-qPCR ( $* P<0.05$ versus TEC-EVs; $n=5$ ). Each experiment was performed in triplicate. 


\begin{tabular}{|c|c|}
\hline keapl & 5' GAGGCCAGGAUGCCUCAGUGUUA \\
\hline miRNA-200a-3p & 3' UGUAGCAAUGGUCUGUCACAAU \\
\hline keapl & 5' GAGGCCAGGAUGCCUCAGUGUUA \\
\hline miRNA-141-3p & 3' GGUAGAAAUGGUCUGUCACAAU \\
\hline
\end{tabular}

Figure S15. Schematic diagram depicting the predicted Keap1 3'-UTR contains one putative miR200a-3p or miR-141-3p binding site. The first eight nucleotides of miR-200a-3p or miR-141-3p are complementary to the binding site in the $3^{\prime}$-UTR. 
Table S1. Primers used in the RT-qPCR assay (mouse)

\begin{tabular}{|c|c|}
\hline Transcription & Primer sequences \\
\hline \multirow{2}{*}{ Keap1 } & Forward: CCCATGAGGCATCACCGTAG \\
\hline & Reverse: CATAGCCTCCGAGGACGTAG \\
\hline \multirow{2}{*}{ Nrf2 } & Forward: TGGACGGGACTATTGAAGGCTG \\
\hline & Reverse: GCCGCCTTTTCAGTAGATGGAGG \\
\hline \multirow{2}{*}{ SOD2 } & Forward: CACATTAACGCGCAGATCATG \\
\hline & Reverse: CCAGAGCCTCGTGGTACTTCTC \\
\hline \multirow{2}{*}{ MT-CO2 } & Forward: CCATAGGGCACCAATGATACTG \\
\hline & Reverse: AGTCGGCCTGGGATGGCATC \\
\hline \multirow{2}{*}{ TNF- $\alpha$} & Forward: CGTCGTAGCAAACCACCAAG \\
\hline & Reverse: TTGAAGAGAACCTGGGAGTAGACA \\
\hline \multirow{2}{*}{ IL-1 $\beta$} & Forward: AAGTGATATTCTCCATGAGCTTTGT \\
\hline & Reverse: TTCTTCTTTGGGTATTGCTTGG \\
\hline \multirow{2}{*}{ IL-8 } & Forward: GCT GGG ATT CAC CTC AAG AA \\
\hline & Reverse: GAA GTG GCA GAA GCT AAC CG \\
\hline \multirow{2}{*}{ IL-6 } & Forward: CAGCAAGATGATCCCAATGA \\
\hline & Reverse: CTCTTGAGCTTGGTGACAA \\
\hline \multirow{2}{*}{$\mathrm{NF}-\kappa \mathrm{B}$} & Forward: CGCCCTCGTCGTGTCGTGA \\
\hline & Reverse: CGGCGCGGGTCTTGTAGTTGC \\
\hline \multirow{2}{*}{ Caspase-9 } & Forward: GGCTGTTAAACCCCTAGACCA \\
\hline & Reverse: TGACGGGTCCAGCTTCACTA \\
\hline \multirow{2}{*}{ GAPDH } & Forward: AGGGCTGCTTTTAACTCTGGT \\
\hline & Reverse: CCCCACTTGATTTTGGAGGGA \\
\hline \multirow{2}{*}{ 18S rRNA } & Forward: CTTAGAGGGACAAGTGGCGTTC \\
\hline & Reverse: CGCTGAGCCAGTCAGTGTAG \\
\hline
\end{tabular}


Table S2. Primers used in the RT-qPCR assay (human)

\begin{tabular}{cl}
\hline Transcription & Primer sequences \\
\hline Keap1 & Forward: TCACCAGGGAAGGATCTACG \\
Nrf2 & Foverse: AGCGGCTCAACAGGTACAGT \\
& Reverse: TCAGCCAGCTTGTCATTTTG \\
SOD2 & Forward: CGCCCTCGTCGTGTCGTGA \\
& Reverse: CGGCGCGGGTCTTGTAGTTGC \\
MT-CO2 & Forward: CAGACGAGGTCAACGATCCCTC \\
& Reverse: GCATGAAACTGTGGTTTGCTCCACAG \\
GAPDH & Forward: CGAGATCCCTCCAAAATCAA \\
& Forward: CTTAGAGGGACAAGTGGCGTTC \\
& Reverse: CGCTGAGCCAGTCAGTGTAG \\
\hline
\end{tabular}


Table S3. Primers used in the RT-qPCR assay (miRNA)

\begin{tabular}{ll}
\hline Reverse transcription primer sequences \\
miR-200a-3p & GTTGGCTCTGGTGCAGGGTCCGAGGTATTCGCACCAG \\
& AGCCAACACATCG \\
miR-141-3p & GTTGGCTCTGGTGCAGGGTCCGAGGTATTCGCACCAG \\
& AGCCAACCATC \\
U6 & GTTGGCTCTGGTGCAGGGTCCGAGGTATTCGCACCAG \\
& AGCCAACGAATTTGC \\
\hline Transcription & primer sequences \\
miR-200a-3p & Forward: TAACACTGTCTGGTAAC \\
& Reverse: GTGCAGGGTCCGAGGT \\
miR-141-3p & Forward: TAACACTGTCTGGTAAAG \\
& Reverse: GTGCAGGGTCCGAGGT \\
& Forward: CCTGCTTCGGCAGCACA \\
\hline U6 & Reverse: GTGCAGGGTCCGAGGT \\
\hline
\end{tabular}

